\section{A touchy subject}

Neural pathways for processing tactile information are arranged so that spatial relationships between receptors on the body surface are reflected in the brain - the organization of sensory cortex conserves the topographic arrangement of the sensory apparatus. Opinion is divided, however, over the role of sensory cortex in perceptual learning and memory. Experiments in rats support the idea that tactile information is also stored in 'early' topographically organized cortex in rats trained for a task with a single whisker, the degree to which learning transferred to other whiskers was determined by the distance between the trained and tested whiskers and by the extent of overlap between the representations of those whiskers in the cortex. But what about humans? Several studies have failed to show that learning of tactile information is topographically organized, suggesting that learned information resides in 'late' cortical areas whose organization is not related to that of the sensory apparatus. By extensively examining the spread of learned tactile information across the fingertips, Harris et al. have now provided evidence to the contrary.

Subjects were trained to distinguish between two stimuli of a par-

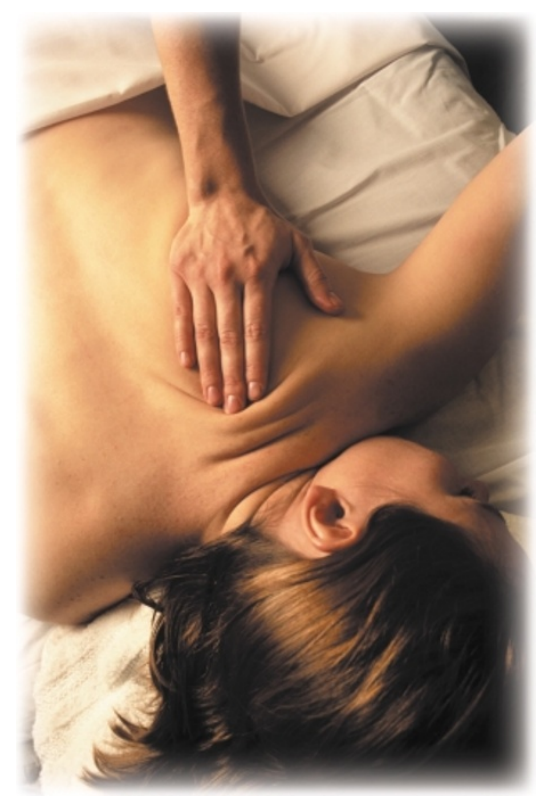
experiments. ticular type with a single finger. Discriminatory ability was then tested with the trained finger, its immediate and second neighbours and the corresponding fingers on the opposite hand to evaluate any transfer of learning. As different types of tactile stimulus are processed in different cortical areas, each with a particular topographic organization, it is possible that any spatial distribution of tactile learning will also vary with stimulus type. To assess this, Harris and colleagues used three types of tactile stimulus: low-frequency vibration, punctate pressure and roughness.

For the vibratory stimulus, learning was confined to the trained fingertip, whereas for both pressure and roughness stimuli, learning with one finger was actually transferred to other, untrained fingers. Transfer of learning depended on topographic distance: performance was good with the immediate neighbour of the trained tip and the corresponding finger on the opposite hand but not with the other fingers tested. It thus seems that - as in rats - learned information is stored in topographically organized early cortical areas, and furthermore, that information is differentially distributed depending on stimulus type.

So, these findings also shed light on the functional organization of the underlying sensory pathways. Using the patterns of transfer for different stimuli and previous observations, the authors suggest the identity of the cortical areas involved in storing information about each stimulus type - Brodmann's area 3b for vibration and area 43 (secondary somatosensory cortex) for punctate pressure and roughness, predictions that could now be tested with further psychophysical

Peter Kirkpatrick

(2) References and links ORIGINAL RESEARCH PAPER Harris, J. A. etal. The topography of tactile learning in humans. J. Neurosci. 21, 1056-1061 (2001) FURTHER READING Harris, J. A. et al. Distribution of tactile learning and its neural basis. Proc. Natl Acad. Sci. USA 96, 7587-7591 (1999) ENCYCLOPEDIA OF LIFE SCIENCES TOUCh

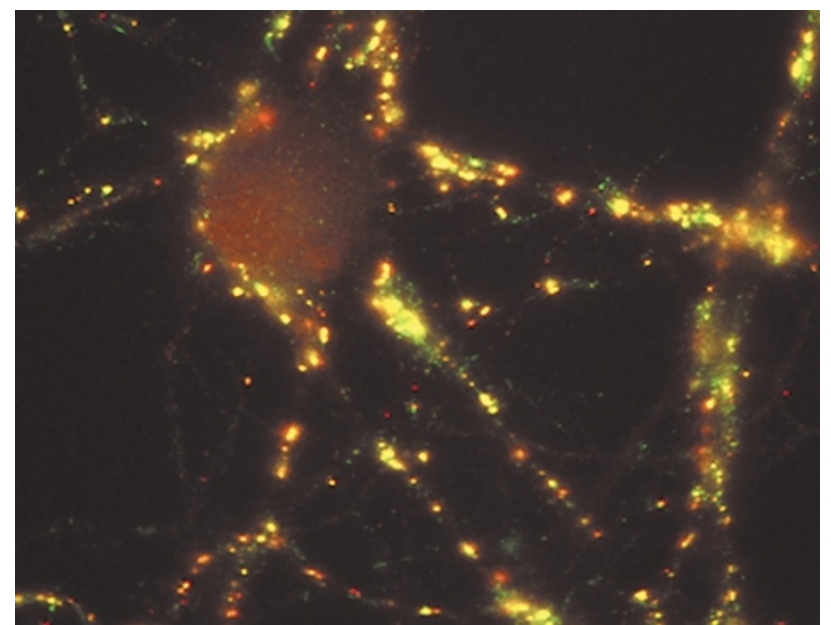

An RGC cultured in the presence of glia, stained for the presynaptic marker synaptotagmin (green) and the postsynaptic marker PSD-95. Courtesy of Karen Christopherson, Stanford University School of Medicine, California, USA.

\section{SYNAPTOGENESIS}

\section{Life support for synapses}

Astrocytes are highly abundant in the brain, but surprisingly little is known about their function. For a long time it was thought that they acted as support cells, and that their chief role was to mop up ions and neurotransmitters at the synaptic cleft. However, as Phil Haydon pointed out in the March issue of Nature Reviews Neuroscience, it is becoming increasingly apparent that their functional relationship with synapses is actually much more intimate and complex. As reported in Science, Ullian et al. have fuelled this discussion by showing that astrocytes play an active role in synapse formation and maintenance.

Formation of synapses in the absence of glia has proved difficult to study in vitro, as many types of neuron depend on glial cells for their survival. However, Ullian et al. have developed a system for culturing purified retinal ganglion cells (RGCs) in a defined serum-free medium. They used this system to compare synapse formation in the presence and absence of astrocytes isolated from the superior colliculus - one of the targets of RGC axons in vivo. They measured the effects of these astrocytes on synapse number, structure, function and stability. In the presence of astrocytes, the average number of synapses per neuron increased sevenfold, although there was no effect on the ultrastructure of individual synapses. Astrocytes also enhanced the efficiency of synaptic transmission, exerting their effects both pre- and postsynaptically. Removal of astrocytes from the cultures led to a fourfold decrease in synapse number over a period of 6 days, providing evidence for a role in synapse stabilization.

So, astrocytes are clearly important for synapse formation and maintenance in vitro, but do they perform the same function in $v i v o$ ? There is certainly some circumstantial evidence for this. During development, for example, RGCs form few synapses in the superior colliculus before the appearance of astrocytes. The observation that astrocytes influence the stability of synapses supports the idea (also reviewed by Haydon) that these cells might also be involved in the regulation of synaptic plasticity. Therefore, it seems that astrocytes deserve a promotion, from humble synaptic cleaner to the level of personal assistant at the very least.

(D) References and links Heather Wood ORIGINAL RESEARCH PAPER Ullian, E. M. et al. Control of synapse number by glia. Science 291, 657-661 (2001)

FURTHER READING Pfrieger, F. W. \& Barres, B. A. New views on synapse-glia interactions. Curr. Opin. Neurobiol. 6, 615-621 (1996) | Haydon, P. G. Glia: listening and talking to the synapse. Nature Rev. Neurosci. 2, 185-193 (2001)

ENCYCLOPEDIA OF LIFE SCIENCES Synapse formation 